\title{
Comparative Analysis of Training Strategies for Neural Network-Based Spectral Unmixing of Laboratory-Simulated Forest Hyperspectral Scenes
}

\author{
Javier Plaza and Antonio Plaza \\ Hyperspectral Computing Laboratory \\ Department of Technology of Computers and Communications \\ University of Extremadura, Avda. de la Universidad s/n, E-10071 Cáceres, SPAIN \\ URL: http://www.umbc.edu/rssipl/people/aplaza-E-mail: aplaza@unex.es
}

\begin{abstract}
In this work, we address the use of neural networks for nonlinear mixture modeling of hyperspectral data by focusing on different training strategies which can automatically generate mixed training samples without a priori information. The proposed approach is compared to the standard, fully constrained linear mixture model using a database of laboratory-simulated forest scenes acquired by the Compact Airborne Spectrographic System (CASI), in which the areal fractions of the main constituents were calculated by the SPRINT canopy model. Our experiments demonstrate that simple multilayer perceptron $(M L P)$ neural networks, when trained using a few mixed training samples, can provide good mixture characterization in different types of vegetation environments.
\end{abstract}

\section{Introduction}

Spectral mixture analysis (also called spectral unmixing) has been an alluring exploitation goal from the earliest days of hyperspectral imaging [7] to our days [12]. No matter the spatial resolution, the spectral signatures collected in natural environments are invariably a mixture of the signatures of the various materials found within the spatial extent of the ground instantaneous field view of the imaging instrument [1]. A standard technique for spectral mixture analysis is linear spectral unmixing [13], which assumes that the collected spectra at the spectrometer can be expressed in the form of a linear combination of endmembers weighted by their corresponding fractional abundances [see Fig. 1(a)]. Although the linear model has some practical advantages, nonlinear spectral unmixing may best characterize the resultant mixed spectra part of the source radiation is multiply scattered before being collected at the sensor [see Fig. 1(b)].

Let us assume that a remotely sensed hyperspectral scene with $n$ bands is denoted by $\mathbf{I}$, in which a pixel is represented by a vector $\mathbf{X}=\left[x_{1}, x_{2}, \cdots, x_{n}\right] \in \Re^{n}$, where $\Re$ denotes the set of real numbers in which the pixel's spectral response $x_{k}$ at sensor channels $k=1, \ldots, n$ is included. Under the nonlinear mixture model assumption, each pixel vector in the original scene can be modeled using the following expression:

$$
\mathbf{X}=f(\mathbf{E}, \boldsymbol{a})+\boldsymbol{n},
$$

where $\mathbf{E}=\left\{\mathbf{e}_{i}\right\}_{i=1}^{p}$ is a matrix of $p$ endmember signatures, $\boldsymbol{a}$ is a $p$-dimensional vector containing the abundances of the endmembers in $\mathbf{X}$, and $f$ is an unknown nonlinear function that defines the interaction between $\mathbf{E}$ and $\boldsymbol{a}$. Neural network techniques have demonstrated great potential to estimate $f$ due to their inherent capacity to approximate complex functions [3]. For decomposing mixed pixels in terms of nonlinear relationships, feed-forward networks such as the multi-layer perceptron (MLP), have been used [15, 2, 14, 11].

An important issue is how to train the neural network architecture to learn nonlinear mixtures. Previous work has demonstrated that intelligent training sample selection algorithms have the potential to optimize the compromise between estimation accuracy and groundtruth knowledge [15]. However, an analysis of the accuracy of such methods in a fully controlled yet realistic scenario is essential. For this purpose, we use a set of laboratory-simulated forest scenes collected using the Compact Airborne Spectrographic Imaging (CASI) system [10]. The Spreading of Photons for Radiation INTerception (SPRINT) canopy model [6] is employed as a physically-inspired reference to evaluate different training sample selection methods.

The remainder of this paper is organized as follows. 


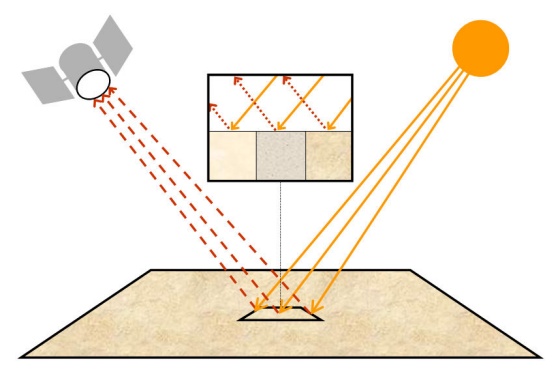

(a)

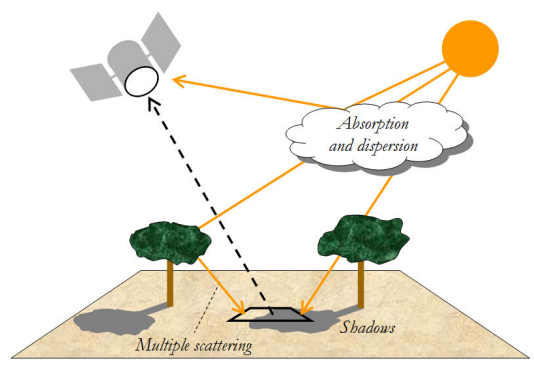

(b)

Figure 1. Linear versus nonlinear mixture models: single (a) versus multiple (b) scattering.

Section 2 describes the considered neural network architecture and training algorithms. Section 3 describes the laboratory-simulated forest scenes. Section 4 provides an experimental assessment of the different considered training strategies. Section 5 concludes with some summarizing points.

\section{Spectral unmixing architecture}

The number of input neurons of the considered MLP architecture equals the number of bands $n$. The number of neurons at the output layer, $p$, equals the number of estimated endmembers, estimated using the concept of virtual dimensionality (VD) [5]. The number of hidden neurons can be fine-tuned depending on the problem under consideration [3]. In this work we are mainly interested in exploring training mechanisms, and thus we empirically set this number to the square root of the product of the number of input features and information classes [11]. Three training strategies are used:

1. Orthogonal subspace projections (OSP) [8]. In this case, it is assumed that $p$ training samples are extracted which are labeled by assuming that the vector of fractional abundances associated to each extracted sample $\boldsymbol{t}_{j}$ is formed by $\hat{a}_{j}=1.0$ (meaning $100 \%$ abundance), and $\hat{a}_{i}=0.0$ (meaning $0 \%$ abundance) for $i \neq j$, with $1 \leq i \leq p$.

2. Unsupervised fully constrained least squares (UFCLS) [4]. This method can be used not only to select but also to label $t$ training samples without assuming prior knowledge about the abundance fractions of endmembers in the input scene, by estimating fractional abundances using a least squares criterion [9] for each training sample selected during the process.

3. Mixed training algorithm (MTA) [14]. This algorithm first extracts $p$ endmembers (in this work, using the OSP). Then, it iteratively seeks for the $t$ most highly mixed pixels in the input data set by following a procedure which behaves in an opposite way as OSP, i.e. it automatically selects and labels a mixed training sample $\boldsymbol{t}_{j}$ by obtaining a vector of abundances $\left[\hat{a}_{1}, \hat{a}_{2}, \cdots, \hat{a}_{p}\right]$ as follows (SA is the spectral angle [4]):

$$
\hat{a}_{i}=\frac{\mathrm{SA}\left(\boldsymbol{t}_{j}, \boldsymbol{e}_{i}\right)}{\sum_{i=1}^{p} \mathrm{SA}\left(\boldsymbol{t}_{j}, \boldsymbol{e}_{i}\right)}, \text { with } 1 \leq i \leq p .
$$

\section{Laboratory-simulated forest scenes}

The CASI scenes used in experiments were obtained by the following procedure [10]. Two kinds of objects, opaque and translucent, were mounted on stems to simulate forest crowns on trunks. These simulated "trees" were randomly placed on a mounting board covered with one of three backgrounds: dark, green, and white. The dimensions of tree crowns for opaque and translucent trees ranged from $1.3 \mathrm{~cm}$ to $1.7 \mathrm{~cm}$. Canopies of both opaque and translucent trees were designed with sparse and dense populations. For the sparse population, 40 trees were planted in an area of $40 \mathrm{~cm}$ by $40 \mathrm{~cm}$, while 100 trees were planted in the same area for the dense population. The scene illumination in this simulation was generated using a $100 \mathrm{~W}$ tungsten lamp and illumination angle of 40 degrees. Twelve hyperspectral images (labeled as CASI01_01 to CASI01_12) were acquired by CASI [10], a pushbroom imager, by moving the entire scene perpendicularly at a constant rate with respect to the CASI field-of view. The scenes (see Fig. 2) were acquired in 72 spectral channels covering the spectral region from 414 to $914 \mathrm{~nm}$, at nominal spectral resolution of $7.5 \mathrm{~nm}$.

\section{Experimental results}

We first applied the standard fully constrained linear linear spectral unmixing (FCLSU) model [9] using the $p=3$ endmembers (sunlit tree, background 


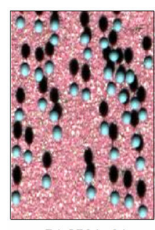

CASI01_01

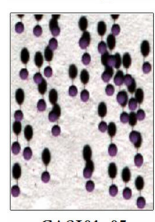

CASI01_05

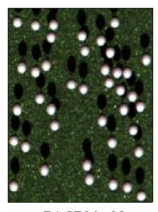

CASI01_09

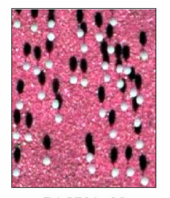

CASI01_02
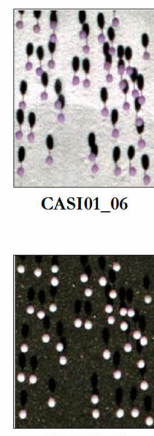

CASI01_10

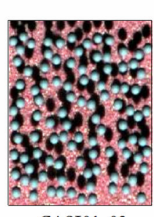

CASI01_03

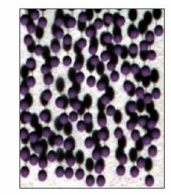

CASI01_07

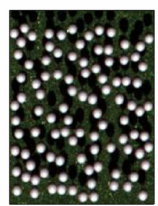

CASI01_11

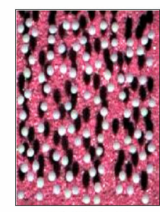

CASI01_04

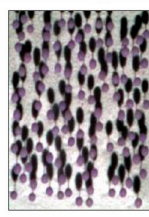
CASI01_08

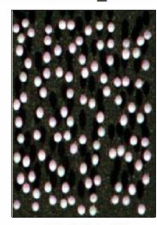

CASI01_12
Figure 2. CASI laboratory scenes.

and shadow) provided by the OSP method. In addition to FCLSU, we also trained the MLP neural network approach using the three considered training strategies (referred to hereinafter as OSP/MLP, UFCLS/MLP and MTA/MLP, where $t=6$ training samples were used in the last two cases). The results obtained were compared with the areal fractions obtained by the SPRINT model.

Table 1 shows the fractional covers estimated for the scenes in Fig. 2. For the scenes with opaque trees and sparse population (01, 05 and 09), the errors in the fractions of the endmembers obtained by FCLSU are larger for the scene with a white background than for the scenes with a dark and green background. For the scene with a white background, the high reflectance of the background increases the multiple scattering between tree crowns and the background, and the reflectance spectrum of the scene departs significantly from a linear mixture of the reflectance spectra of sunlit tree, background, and shadow. When the UFCLS and MTA algorithms were used for training, the estimation results were significantly increased with regards to all other tested methods for the scene with white background.

On the other hand, the fractions of the endmembers obtained for the scenes with sparse translucent tree crowns $(02,06$ and 10$)$ were the most similar to those reported by the SPRINT model for the scenes with sparse translucent trees. Comparison of FCLSU-based estimation results for the translucent cases with those for the opaque cases reveals that the errors in the fractions of sunlit tree, background and shadow obtained by FCLSU are generally larger with translucent crowns. This is likely due to the relative increase in multiple scattering between crowns and the background due to the crown transparency.

Considering the scenes with opaque trees and dense canopies (03, 07 and 11), the FCLSU estimation errors for the scene with white background are increased compared with the scenes with a dark and green background, due to the large multiple scattering between tree crowns and the white background. Comparing with the results for the scenes with sparse canopies, we observe that the errors in the FCLSU-estimated fractions of sunlit tree, background, and shadow obtained by the linear unmixing are larger for the scenes with dense opaque crowns. This is because, for the dense canopies, multiple scattering effects are more significant.

Finally, the errors in the fractions of the endmembers for the scenes with translucent trees and dense population $(04,08$ and 12) are larger for the scene with a white background. Compared with the results obtained for the scenes with opaque crowns, the errors in the fractions of sunlit tree, background, and shadow are increased. Further, the errors in the FCLSU-estimated fractions for the scenes with dense canopies are larger than those obtained for the scenes with sparse canopies. In this scenario, the MLP trained using mixed labeled samples provided by UFCLS seemed to perform appropriately for the scenes with green and dark background and also for the scenes with white background.

\section{Conclusions and summary}

In this paper, we have reported the use of laboratorysimulated scenes to investigate the effects of background reflectance, crown transparency, and stem density on the spectral mixing systematics expected in a forest stand. The SPRINT canopy model was used to evaluate the abundance fractions provided by different spectral mixture analysis procedures (linear and nonlinear neural network-based). We observed that, for the scenes with a white background, the multiple scattering between crowns and background are quite large. If linear unmixing is used for these scenes, the errors in the fractions of the endmembers are quite large. In addition, the errors in the fractions are larger for dense canopies than for sparse canopies, and they are also larger for translucent crowns than for opaque crowns. This is because both the stem density and the crown transparency increase multiple scattering between crowns and the background. In order to model this phenomenon, we have experimentally shown that a simple neural network-based model with intelligent training can significantly improve the results provided by the linear mixture model in a fully controlled analysis scenario with known fractional abundances. 


\begin{tabular}{|c|c|c|c|c|c|c|c|c|c|}
\hline & \multicolumn{3}{|c|}{ CASI01_01 (Green background) } & \multicolumn{3}{|c|}{ CASI01 09 (Dark background) } & \multicolumn{3}{|c|}{ CASI01 05 (White background) } \\
\hline SMA method & Sunlit tree & Background & Shadow & Sunlit tree & Background & Shadow & Sunlit tree & Background & Shadow \\
\hline FCLSU & 0.083 & 0.727 & 0.189 & 0.129 & 0.737 & 0.136 & 0.074 & 0.655 & 0.270 \\
\hline OSP/MLP & 0.095 & 0.724 & 0.180 & 0.097 & 0.729 & 0.153 & 0.127 & 0.609 & 0.264 \\
\hline UFCLS/MLP & 0.108 & 0.709 & 0.183 & 0.101 & 0.797 & 0.102 & 0.106 & 0.707 & 0.186 \\
\hline MTA/MLP & 0.105 & 0.735 & 0.166 & 0.110 & 0.734 & 0.154 & 0.115 & 0.710 & 0.173 \\
\hline \multirow[t]{2}{*}{ SPRINT } & 0.109 & 0.723 & 0.167 & 0.109 & 0.723 & 0.167 & 0.109 & 0.723 & 0.167 \\
\hline & \multicolumn{3}{|c|}{ CASI01_02 (Green background) } & \multicolumn{3}{|c|}{ CASI01_10 (Dark background) } & \multicolumn{3}{|c|}{ CASI01_06 (White background) } \\
\hline SMA me & Sunlit tree & Background & Shadow & Sunlit tree & Background & Shadow & Sunlit tree & Background & Shadow \\
\hline FCLSU & 0.074 & 0.805 & 0.120 & 0.081 & 0.813 & 0.104 & 0.105 & 0.796 & 0.099 \\
\hline MLP & 0.073 & 0.746 & 0.180 & 0.093 & 0.8 & 0.103 & 0.129 & 0.464 & 0.407 \\
\hline UFCLS/MLP & 0.099 & 0.769 & 0.133 & 0.119 & 0.707 & 0.172 & 0.104 & 0.665 & 0.228 \\
\hline MTA/MLP & 0.086 & 0.722 & 0.191 & 0.119 & 0.738 & 0.142 & 0,09 & 0,737 & 0,172 \\
\hline \multirow[t]{2}{*}{ SPRINT } & 0.104 & 0.701 & 0.194 & 0.104 & 0.701 & 0.194 & 0.104 & 0.701 & 0.194 \\
\hline & \multicolumn{3}{|c|}{ CASI01_03 (Green background) } & \multicolumn{3}{|c|}{ CASI01_011 (Dark background) } & \multicolumn{3}{|c|}{ CASI01_07 (White background) } \\
\hline SMA method & Sunlit tree & Background & Shadow & Sunlit tree & Background & Shadow & Sunlit tree & Background & Shadow \\
\hline FCLSU & 0.183 & 0.404 & 0.412 & 0.347 & 0.390 & 0.258 & 0.204 & 0.427 & 0.367 \\
\hline OSP/MLP & 0.232 & 0.457 & 0.311 & 0.261 & 0.388 & 0.291 & 0.315 & 0.445 & 0.240 \\
\hline UFCLS/MLP & 0.255 & 0.388 & 0.357 & 0.272 & 0.422 & 0.194 & 0.287 & 0.456 & 0.257 \\
\hline MTA/MLP & 0.283 & 0.409 & 0.307 & 0.296 & 0.386 & 0.293 & 0.290 & 0.377 & 0.335 \\
\hline \multirow[t]{2}{*}{ SPRINT } & 0.294 & 0.383 & 0.318 & 0.294 & 0.383 & 0.318 & 0.294 & 0.383 & 0.318 \\
\hline & \multicolumn{3}{|c|}{ CASI01_04 (Green background) } & \multicolumn{3}{|c|}{ CASI01_012 (Dark background) } & \multicolumn{3}{|c|}{ CASI01_08 (White background) } \\
\hline SMA method & Sunlit tree & Background & Shadow & Sunlit tree & Background & Shadow & Sunlit tree & Background & Shadow \\
\hline FCLSU & 0.180 & 0.547 & 0.272 & 0.167 & 0.507 & 0.326 & 0.249 & 0.491 & 0.260 \\
\hline OSP/MLP & 0.178 & 0.567 & 0.253 & 0.151 & 0.657 & 0.191 & 0.208 & 0.531 & 0.259 \\
\hline UFCLS/MLP & 0.371 & 0.385 & 0.242 & 0.137 & 0.668 & 0.194 & 0.287 & 0.456 & 0.257 \\
\hline MTA/MLP & 0,264 & 0,349 & 0,387 & 0.253 & 0.371 & 0.387 & 0.253 & 0.367 & 0.379 \\
\hline SPRINT & 0.279 & 0.357 & 0.362 & 0.279 & 0.357 & 0.362 & 0.279 & 0.357 & 0.362 \\
\hline
\end{tabular}

Table 1. Fractional abundance estimation results for the laboratory-simulated forest scenes.

\section{References}

[1] J. B. Adams, M. O. Smith, and P. E. Johnson. Spectral mixture modeling: a new analysis of rock and soil types at the Viking Lander 1 site. Journal of Geophysical Research, 91:8098-8112, 1986.

[2] A. Baraldi, E. Binaghi, P. Blonda, P. A. Brivio, and P. Rampini. Comparison of the multilayer perceptron with neuro-fuzzy techniques in the estimation of cover class mixture in remotely sensed data. IEEE Transactions on Geoscience and Remote Sensing, 39:994-1005, 2001.

[3] C. M. Bishop. Neural networks for pattern recognition. Oxford: Oxford University Press, 1995.

[4] C.-I. Chang. Hyperspectral Imaging: Techniques for Spectral Detection and Classification. Kluwer Academic/Plenum Publishers: New York, 2003.

[5] C.-I. Chang and Q. Du. Estimation of number of spectrally distinct signal sources in hyperspectral imagery. IEEE Transactions on Geoscience and Remote Sensing, 42(3):608-619, 2004

[6] N. S. Goel and R. L. Thompson. A snapshot of canopy reflectance models and a universal model for the radiation regime. Remote Sensing Reviews, 18:197-225, 2000.

[7] A. F. H. Goetz, G. Vane, J. E. Solomon, and B. N. Rock. Imaging spectrometry for Earth remote sensing. Science, 228:1147-1153, 1985.

[8] J. C. Harsanyi and C.-I. Chang. Hyperspectral image classification and dimensionality reduction: An orthogonal subspace projection. IEEE Transactions on Geoscience and Remote Sensing, 32(4):779-785.
[9] D. Heinz and C.-I. Chang. Fully constrained least squares linear mixture analysis for material quantification in hyperspectral imagery. IEEE Transactions on Geoscience and Remote Sensing, 39:529-545, 2001.

[10] B. Hu, J. R. Miller, J. Wang, and N. S. Goel. Investigation of linear spectral mixtures of the reflectance spectra using laboratory simulated forest scenes. Proc. IEEE Intl. Geosci. Remote Sens. Symp., 3:1535-1537, 2002.

[11] W. Liu and E. Y. Wu. Comparison of non-linear mixture models. Remote Sensing of Environment, 18:19762003, 2004.

[12] A. Plaza, J. A. Benediktsson, J. Boardman, J. Brazile, L. Bruzzone, G. Camps-Valls, J. Chanussot, M. Fauvel, P. Gamba, J. Gualtieri, M. Marconcini, J. C. Tilton, and G. Trianni. Recent advances in techniques for hyperspectral image processing. Remote Sensing of Environment, 113:110-122, 2009.

[13] A. Plaza, P. Martinez, R. Perez, and J. Plaza. A quantitative and comparative analysis of endmember extraction algorithms from hyperspectral data. IEEE Transactions on Geoscience and Remote Sensing, 42(3):650663, 2004.

[14] J. Plaza and A. Plaza. Spectral mixture analysis of hyperspectral scenes using intelligently selected training samples. IEEE Geoscience and Remote Sensing Letters, 7, 2010, doi: 10.1109/LGRS.2009.2036139.

[15] J. Plaza, A. Plaza, R. Perez, and P. Martinez. On the use of small training sets for neural network-based characterization of mixed pixels in remotely sensed hyperspectral images. Pattern Recognition, 42:3032-3045, 2009. 